e-Journal of Educational

Research, Assessment and Evaluation

\section{REIIEVE}

Revista ELectrónica de Investigación y EValuación Educativa

\title{
TENDENCIAS EN LA NORMALIZACIÓN DE NOMBRES DE AUTORES EN PUBLICACIONES CIENTÍFICAS
}

\section{[Normalization trends of authors' names in scientific publications]}

\author{
by/por
}

$\underline{\text { Article record }}$

About authors

$\underline{\text { HTML format }}$

Aliaga, Francisco M. (Francisco.Aliaga@uv.es)

Correa, Ana D. (acorrea@ull.es)
Ficha del artículo

$\underline{\text { Sobre los autores }}$

Formato HTML

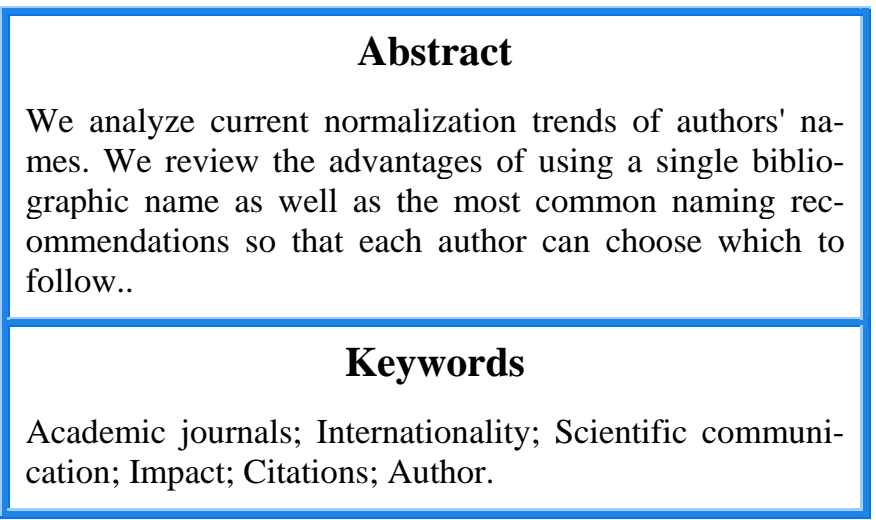

Los últimos años venimos asistiendo a un incremento sustancial en la internacionalización de la producción académica. Esto es particularmente cierto en el ámbito español $\mathrm{y}$, específicamente, en las revistas de educación (Aliaga y Suárez-Rodríguez, 2007; Hernández Pina y Maquilón, 2010). Son muchos y variados los factores que influyen en esta mayor preocupación por la dimensión internacional de nuestras investigaciones, pero cabría destacar entre ellos:

- La normativa para la evaluación de la investigación del personal científico (ANECA, CNEAI, etc. en España o de instituciones como CONACYT en México, CAICYT en Argentina o Colciencias en Co- lombia, por citar sólo algunas del ámbito latinoamericano).

- Diversos impulsos y orientaciones para favorecer la mejora y profesionalización de la edición científica, con ayudas prácticas como los textos de Román (1981) o el de Delgado López-Cózar, Ruiz Pérez y Jiménez Contreras, (2007), éste último dentro del proyecto ARCE (Apoyo a las Revistas Científicas Españolas), de la Fundación Española para la Ciencia y la Tecnología (FECYT).

- Desarrollo de nuevas herramientas de evaluación de calidad y/o impacto, como Latindex o In-RECS.

- Nacimiento e impulso de hemerotecas virtuales y directorios, orientados a facili- 
tar al lector la consulta simultánea de una gran cantidad de revistas (citaremos proyectos tan interesantes como Redalyc, erevistas, DOAJ, etc.).

- Las negociaciones de la FECYT respecto a la gestión unificada de las relaciones con bases de datos ISI-Thompson.

En este contexto se va incrementando notablemente el acceso de revistas a bases de datos internacionales, incluso de revistas (como las hispanas) con un interés predominantemente regional (Rodríguez Yunta, 2010). Por un lado, se está produciendo una acelerada incorporación a las bases de datos del Web of Knowledge (ISI-ThompsonReuters), que tradicionalmente había ignorado las producciones en Ciencias Sociales de ámbitos culturales no anglosajones. La progresiva mercantilización del Institute of Scientific Information creado por Garfield, así como una creciente complejidad en ese mercado con la aparición de iniciativas competidoras, ha modificado sustancialmente la política comercial -afectando con ello a la científica- de dicha institución. Por otro lado, asistimos también a la progresiva implantación de algunas de las alternativas emergentes (SCOPUS, Scholar Google), que incluyen un número de revistas sensiblemente superior a las de su competidora del Web of Knowledge. Estas y otras bases de datos internacionales, la mayoría de origen anglosajón, son un elemento clave para propiciar la diseminación del conocimiento, fomentando así la difusión de las propias revistas y de los autores. Son, por tanto, un elemento esencial para incrementar el impacto medido de la actividad científica.

Sin embargo, la relativa falta de experiencia de muchos académicos en este nuevo contexto internacional propicia algunos problemas que afectan a la visibilidad de su trabajo. A ello hay que añadir las dificultades debidas a las diferencias culturales entre los creadores de las principales bases de datos (como hemos dicho, fundamentalmente anglosajones) y los diversos contextos naciona- les a los que se proyectan como expresión de su globalización. Se produce así una gran cantidad de desencuentros y errores que afectan a la calidad y eficacia de los procesos de codificación y recuperación de la información.

Una de las principales fuentes de estos errores es la idiosincrasia de cada país en la manera de expresar los nombres de los autores. Como es bien sabido, para nombrar a las personas los anglosajones (y muchos otros países europeos) utilizan predominantemente el apellido del padre exclusivamente, junto con un nombre, o en ocasiones varios, individuales o 'de pila'. Este sistema, aunque propicia las coincidencias (y, por tanto, la confusión de identidades) es relativamente sencillo de utilizar, ya que el apellido o denominación familiar se identifica fácilmente en cualquier texto: sólo hay que buscar la última palabra (last name) del nombre. Adicionalmente, provoca algunos problemas para la identificación de los autores debido a la costumbre, ligeramente en retroceso, de que la esposa adopte el apellido del marido, generalmente perdiendo el propio (Goldin \& Shim, 2004).

Otras culturas utilizan formas específicas y diferentes de identificar a las personas, que implican complicaciones no consideradas (o resueltas) con el sistema al que hemos hecho referencia anteriormente y que hemos dado en llamar, por simplificar, anglosajón. Así, en el caso de países como Corea, un 22\% de sus ciudadanos se apellidan Kim (también como apellido único), por lo que no es nada raro encontrar coincidencia de denominaciones entre cientos e incluso miles de personas (según datos de 2004 de la National Statistical Office de la República de Corea). En el caso de China, prestigiosas revistas como Nature han señalado (Qiu, 2008), alarmadas, los graves problemas de identificación derivados del sistema utilizado. Así, el 85\% de la población china (unos 1.100 millones de personas) comparten los apenas 129 apellidos más comunes, lo que da una ligera idea 
de la enorme cantidad de coincidencias producidas (cfr. Qiu, 2008). A esto, entre otros problemas se añade la variación nombreapellido o apellido-nombre según diversas costumbres, así como las separaciones silábicas (o no) de los nombres. Otros problemas relacionados se dan en zonas como el sur de la India, donde casi no se usan apellidos y, como sustituto, tiende a utilizarse el nombre del padre, o en los países eslavos, donde los hijos usan un patronímico, que toman del nombre del padre, al que se le añade una desinencia.

En el caso de la denominación hispánica o ibérica, que incluye también a los países de cultura portuguesa, en la mayoría de casos se hereda tanto el apellido del padre como el de la madre, además de tener uno o, frecuentemente, varios nombres personales o de pila. Este sistema provoca frecuentes confusiones cuando se incluye a los autores en bases de datos creadas con una mentalidad 'anglosajona', ya que entonces se toma el último término como apellido identificador del sujeto, por el hábito de buscar el 'last name' e identificarlo con su apellido, que en nuestro caso no es el de uso habitual, sino el materno o secundario. Por ello es frecuente que un mismo texto parezca tener distinto autor dependiendo de si lo buscamos en bases de datos 'hispanas' o 'anglosajonas', ya que en aquellas se usa el primer apellido para identificar a los autores y en éstas el segundo.

Para que podamos calibrar la importancia de este tema, recordaremos que diversos estudios (Costas y Bordons, 2007; Ruiz-Pérez, López-Cózar y Jiménez-Contreras, 2002) han encontrado que en las bases de datos de más amplia difusión internacional, las denominadas ISI-Thompsom-Reuters (SCI, SSCI y AHCI), el porcentaje de autores españoles que aparecen con dos o más nombres distintos varía entre un $20 \%$ y un $40 \%$, dificultando así tanto su localización como el cálculo del impacto de su obra (por ejemplo, para calcular valores como el índice $H$ ).
En consecuencia, elegir cómo se firman los artículos es algo que debería meditarse cuidadosamente y con fundamento, porque de esa decisión de utilizar un "Nombre Bibliográfico Único", dependerá la visibilidad e identificabilidad de los autores, un elemento muy importante en la difusión de su trabajo y en el reconocimiento de sus méritos. La necesidad de formalizar adecuadamente el nombre con el que se firma -lo que antiguamente se denominaba 'nombre de pluma'implica, por tanto, consecuencias relevantes para los autores. Tan importante es el tema, que la Fundación Española de Ciencia y Tecnología encargó al grupo EC3 encargado del ranking InRECS- un manual con recomendaciones "de ayuda a los investigadores españoles para la normalización del nombre de autores e instituciones en las publicaciones científicas" (FECYT, 2007). Estas recomendaciones son aplicables a todos aquellos autores que utilizan un nombre al estilo que hemos denominado hispánico o ibérico, mayoritario en los países latinoamericanos.

Desde la revista RELIEVE nos decantamos por apoyar las recomendaciones del manual de la FECYT (2007), en un intento de favorecer la adecuada indexación y un mayor impacto de nuestros autores y de nuestra publicación, aunque siempre respetaremos la voluntad expresada finalmente por los autores.

Hemos de hacer, para empezar, una primera observación que, por evidente, no sería necesario resaltar si no fuera porque se incumple con demasiada frecuencia. Se trata de que los investigadores no tienen que usar, necesariamente, el nombre completo y exacto que aparece en los registros civiles. Puede optarse entre usar uno o los dos apellidos, como se hace frecuentemente en la vida cotidiana, según el uso que se vaya a hacer del nombre. También puede usarse uno de los nombres de pila o varios. La finalidad es que nos identifiquemos adecuadamente, no hacer una copia fiel de la partida de nacimiento. 
Hay que tener bien presente que el nombre que utilicemos será procesado por distintas bases de datos (hispanas, anglosajonas y de otro tipo), con procedimientos distintos y con resultados potencialmente muy diferentes unos de otros.

Veamos algunas recomendaciones básicas respecto al nombre que deberían usar los autores hispanos según el citado manual de la FECYT (2007):

1. Firmar siempre todos los trabajos científicos y/o académicos con el mismo nombre. Es demasiado frecuente ver que un mismo investigador a veces usa un apellido y a veces los dos; y en unas ocasiones emplea los distintos nombres y en otras usa sus iniciales para acortarlo. Esto sólo conlleva confusión y dificultad para localizar la obra producida. Debería inculcarse a todos los investigadores noveles que identificarse apropiadamente conlleva beneficios, tanto personales como para la comunidad académica. Invertir un poco de tiempo en elegir bien ese 'nombre de pluma' al inicio de cualquier carrera es algo que rendirá frutos. Como ejemplo de que muchos somos 'pecadores' que hemos cometido este error tan habitual, mostramos a continuación los distintos nombres con los que aparecen los autores de este texto en una base de datos que suele manejar nombres hispánicos (ISOC - Ciencias Sociales y Humanidades, creada por el Consejo Superior de Investigaciones Científicas español) y otra de origen anglosajón (Scholar Google). Hemos incluido en cursiva aquellas que hemos detectado con erratas.

Tabla 1 - Versiones del nombre de los autores en diversas base de datos

\begin{tabular}{|c|c|c|}
\hline Base de datos & Versiones del nombre & Versiones del nombre \\
\hline ISOC & $\begin{array}{l}\text { Aliaga, Francisco } \\
\text { Aliaga, F. } \\
\text { Aliaga, Francisco M } \\
\text { Aliaga Abad, Francisco M. } \\
\text { Aliaga Abad, Francisco }\end{array}$ & $\begin{array}{l}\text { Correa, Ana Delia } \\
\text { Correa, Ana-Delia } \\
\text { Correa, Ana D. } \\
\text { Correa Piñero, Ana Delia }\end{array}$ \\
\hline Scholar Google & $\begin{array}{l}\text { FA Abad } \\
\text { FMA Abad } \\
\text { F Aliaga } \\
\text { FM Aliaga } \\
\text { F Aliaga Abad } \\
\text { FM Aliaga Abad }\end{array}$ & $\begin{array}{l}\text { AD Correa } \\
\text { AD Correa Piñero } \\
\text { ADC Piñero } \\
\text { ANADC PIÑERO } \\
\text { Correa Piñero, Ana Delia } \\
\text { AD Correa-Piñero } \\
\text { C Piñero } \\
\text { ADC Piñeiro }\end{array}$ \\
\hline
\end{tabular}

2. En general, el formato más recomendable es el de "Nombre + Inicial (opcional) + primer apellido”. Esta opción será la elegida por aquellos autores que no tengan un apellido demasiado frecuente, como García, González, Sánchez..., en cuyo caso el esquema propuesto no cumpliría con el requisito de identificación del autor, ya que podría confundírsele con muchas otras personas con las que comparte un apellido tan habitual.
Respecto a los nombres de pila, hay que evitar que se confunda el segundo nombre (caso de que exista) con uno de los apellidos. Para ello lo más recomendable es alguna de las siguientes opciones:

a. utilizar sólo la inicial,

b. omitir el nombre menos utilizado

c. poner ambos, pero unidos por un guión, para mostrar así la conexión de ambos términos como nombre. Esta opción será la preferible cuando los nombres sean muy habituales y propensos, 
por tanto, a la confusión. También en el

ninguno de los nombres que se tiene.

caso de que no se quiera renunciar a

Tabla 2 - Aplicaciones de las recomendaciones - Apellido distinguible

\begin{tabular}{|c|l|l|}
\hline Recomendación & \multicolumn{1}{|c|}{ Nombre completo } & \multicolumn{1}{c|}{ Recomendación } \\
\hline 2 & $\begin{array}{l}\text { Tomás Escudero Escorza } \\
\text { Eduardo Backhoff Escudero }\end{array}$ & $\begin{array}{l}\text { Tomás Escudero } \\
\text { Eduardo Backhoff }\end{array}$ \\
\hline $2 \mathrm{a}$ & $\begin{array}{l}\text { Francisco Miguel Aliaga Abad } \\
\text { Ana Delia Correa Piñero }\end{array}$ & $\begin{array}{l}\text { Francisco M. Aliaga } \\
\text { Ana D. Correa }\end{array}$ \\
\hline $2 \mathrm{~b}$ & $\begin{array}{l}\text { Francisco Javier Tejedor Tejedor } \\
\text { María Natividad Orellana Alonso }\end{array}$ & $\begin{array}{l}\text { Javier Tejedor } \\
\text { Natividad Orellana }\end{array}$ \\
\hline $2 \mathrm{c}$ & $\begin{array}{l}\text { Ana Delia Correa Piñero } \\
\text { José Antonio Téllez Muñoz }\end{array}$ & $\begin{array}{l}\text { Ana-Delia Correa } \\
\text { José-Antonio Téllez }\end{array}$ \\
\hline
\end{tabular}

3. En el caso de autores con apellidos comunes se optará por utilizar ambos apellidos, para reducir la probabilidad de ser confundido con otras personas de nombre semejante. Sin embargo ha de evitarse que se entienda como apellido sólo la última palabra (last name), por lo que se recomienda que en estos casos se unan ambos apellidos mediante un guión. Esta opción podría usarse también para los autores con un primer apellido no muy común, pero que quieran mantener su identificación más completa o reducir la probabilidad de confusión con otra persona. Para los nombres se mantienen las mismas recomendaciones indicadas en el apartado anterior.

Tabla 3 - Aplicaciones de las recomendaciones- Apellido común

\begin{tabular}{|c|l|l|}
\hline Recomendación & \multicolumn{1}{|c|}{ Nombre completo } & \multicolumn{1}{c|}{ Recomendación } \\
\hline 3 & $\begin{array}{l}\text { Gregorio Rodríguez Gómez } \\
\text { Manuel López Torrijo }\end{array}$ & $\begin{array}{l}\text { Gregorio Rodríguez-Gómez } \\
\text { Manuel López-Torrijo }\end{array}$ \\
\hline 3a & Jesús Modesto Suárez-Rodríguez, & Jesús M. Suárez-Rodríguez \\
\hline 3b & $\begin{array}{l}\text { Víctor Benito Álvarez Rojo } \\
\text { Jesús Modesto Suárez-Rodríguez, }\end{array}$ & $\begin{array}{l}\text { Víctor Álvarez-Rojo } \\
\text { Jesús Suárez-Rodríguez }\end{array}$ \\
\hline 3c & $\begin{array}{l}\text { Miguel Ángel Gómez Ruiz, } \\
\text { Rosario Leticia Dominguez Guedea }\end{array}$ & $\begin{array}{l}\text { Miguel-Ángel Gómez-Ruiz } \\
\text { Rosario-Leticia Dominguez-Guedea }\end{array}$ \\
\hline
\end{tabular}

4. Las partículas gramaticales (artículos, preposiciones) en la denominación de los autores suelen acabar convirtiéndose en un problema ya que algunas bases de datos las respetan en su posición, mientras que otras, al no ser elementos significativos, no las toman en consideración.

a. En los nombres propios: Se recomienda eliminarlas sin más, ya que por el contexto se entiende sobradamente y no añaden singularidad a la denominación. b. En los apellidos: Las partículas provocan cierta confusión, ya que pueden entenderse como parte del apellido o como complemento aparte. Por ello lo mejor sería evitarlas. De todos modos, muchas personas pueden desear mantener su nombre incluyendo ese aspecto que le aporta singularidad. En tal caso debería unir todas las palabras mediante guiones, a fin de constituir una única unidad de referencia. 
Tabla 4 - Aplicaciones de las recomendaciones- Partículas gramaticales

\begin{tabular}{|c|l|l|}
\hline Recomendación & \multicolumn{1}{|c|}{ Nombre completo } & \multicolumn{1}{|c|}{ Recomendación } \\
\hline 4a & $\begin{array}{l}\text { María del Carmen Jiménez Fernández } \\
\text { María del Pilar Sepúlveda-Ruiz }\end{array}$ & $\begin{array}{l}\text { Carmen Jiménez [o Jiménez-Fernández] } \\
\text { María-Pilar Sepúlveda [o Sepúlveda-Ruiz] }\end{array}$ \\
\hline \multirow{2}{*}{ 4b } & $\begin{array}{l}\text { Rakel del Frago Arbizu } \\
\text { Arturo de la Orden Hoz }\end{array}$ & $\begin{array}{l}\text { Rakel Frago o Rakel del-Frago } \\
\text { Arturo de-la-Orden }\end{array}$ \\
\hline
\end{tabular}

5. Abreviaturas: Hay algunas abreviaturas que, por ser muy habituales en nuestro contexto, suelen utilizarse frecuentemente, ya que no normalmente no provocan problemas de comprensión y se entienden bien. Sin embargo, al publicar artículos científicos que pueden ser recogidos en bases de datos internacionales, la condición de 'en nuestro contexto' deja de aplicarse y, por tanto, esas abreviaturas pasan a convertirse en un problema para la correcta identificación de los autores. Por ello deben evitarse, generalmente utilizando la palabra completa o eliminándola. Esta es una fuente de variación muy importante debido a la extensión de su uso, por lo que habrá que poner un cuidado especial en evitarla, escribiendo siempre el nombre de la misma manera en todos los casos.

Tabla 5 - Abreviaturas de nombres

\begin{tabular}{|c|c|c|}
\hline Recomendación & Nombre completo & Recomendación \\
\hline 5 & $\begin{array}{l}\mathbf{M}^{\mathbf{a}} \text { del Carmen Jiménez } \\
\text { M}^{\mathrm{a}} \text { Ángeles Rebollo Catalán }\end{array}$ & $\begin{array}{l}\text { María-del-Carmen Jiménez [o Carmen Jiménez] } \\
\text { Mari-Ángeles Rebollo [o Ángeles Rebollo] }\end{array}$ \\
\hline
\end{tabular}

6. Un último problema a analizar antes de elegir la forma definitiva y estable con el que firmar nuestros trabajos tiene que ver con las tildes. Se trata de un tipo de símbolos que no se usan en inglés, por lo que no es raro que algunas bases de datos los interpreten con códigos extraños, lo que dificulta la localización del autor y sus textos. En otros casos sencillamente se eliminan las tildes y se sustituyen por las letras sin acentuar. A veces todo esto se hace extensivo a nuestra distintiva 'ñ', que o bien se interpreta con códigos extraños o bien se sustituye por una 'n'. Por último, algunas bases de datos (generalmente las hispanas, pero no sólo éstas) pueden respetar los acentos en los nombres. Debido a que escribir en español una palabra sin el acento que le corresponde se suele considerar una falta de ortografía, algunos autores optan por mantener su nombre con los acentos, mientras que otros prefieren no utilizarlos, primando más la proyección de su trabajo.
En cualquier caso, como en todos los demás aspectos que estamos revisando, la regla de oro a tener en cuenta por los autores sería la de mantener siempre el mismo formato a la hora de firmar y que este 'nombre de autor' nos permita identificarnos claramente, evitando confusiones.

Desde la revista RELIEVE optamos, como hemos comentado anteriormente, por respetar la voluntad de cada autor, por lo que a veces puede darse la paradoja de que, en un mismo artículo, un mismo apellido como es el caso de Salmerón, aparece escrito de distinta manera para cada autor (por ejemplo, Salmerón-Pérez, Gutierrez-Braojos, Fernández-Cano \& Salmeron-Vilchez, 2010).

Este aspecto de la estandarización de los nombre de los autores en la literatura científica está resultando tan importante que surgen diversas iniciativas para apoyarlo. Una de ellas es IraLIS (International Registry of Authors-Links to Identify Scientists), una iniciativa española (apoyada por la FECYT) 
cuya finalidad, declarada en su web, se plasma en cinco objetivos:

1. "Concienciar a los autores científicos sobre la importancia de la firma para ser citados correctamente, y para poder recuperar toda la bibliografía a lo largo de su carrera profesional.

2. Redactar criterios de firma normalizada para a) ser indexado correctamente $y$ b) distinguirse de otros autores con nombres iguales.

3. Producir una base de datos con todas las variantes de firma utilizadas por cada autor.

4. Realizar búsquedas bibliográficas automáticas usando las variantes de firma.

5. Ser la autoridad de firmas del repositorio E-LIS" ('E-prints in Library and Information Science', un almacén de docu- mentos sobre biblioteconomía y documentación).

El sistema creado por IraLIS permite que un autor escriba su nombre completo, devolviéndole a continuación un listado de variantes del mismo que cumplirían con los criterios más habituales de estandarización de nombres hispanos. Cada persona puede entonces elegir entre estas opciones la que desee convertir en su firma habitual. Puede registrar ese formato elegido en IraLIS, al que el sistema añadirá las variantes que pueden producir las distintas bases de datos a partir del nombre completo ('oficial') del autor. Eso facilitará enormemente la localización de la producción científica publicada por ese autor concreto. Por ello recomendamos desde estas páginas la inscripción de los autores hispanos en esta iniciativa, tan loable como desinteresada.



Figura 1 - Ejemplo de Ficha IraLIS
Los problemas con la correcta identificación de los autores preocupan no sólo a los propios implicados. También las grandes empresas que gestionan las principales bases de datos bibliográficas quieren reducir el número de errores y desarrollar herramientas que permitan búsquedas más precisas. Así, por ejemplo, las bases de datos conocidas antiguamente como ISI (o incluidas en la Web of Knowledge, de Thompson-Reuters) intentan contribuir a este proceso con el desarrollo y puesta en marcha, desde el año 2008, de ResearchID. Se trata de una inicia- tiva que plantea que cada investigador pase a tener un único número identificador, algo así como un número de un documento de identidad que permita evitar confusiones (desambiguación) y realizar las búsquedas bibliográficas sin confusiones de identidad. Se trata de un tema polémico y poco estructurado (no ha habido intentos de coordinación con otros proyectos similares), pero que dada la potencia de la empresa que lo promueve, bien pudiera llegar a tener ciertas probabilidades de éxito. 


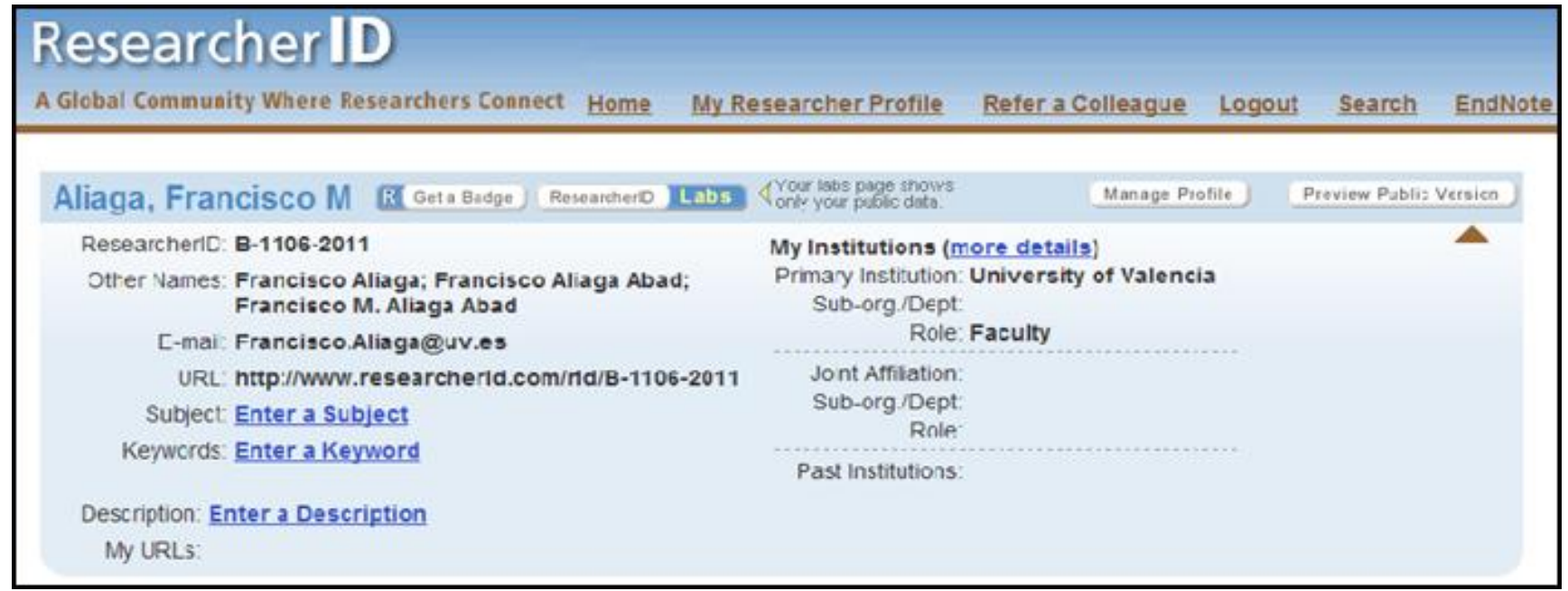

Figura 2 - Ficha de identificación y asignación de número en Researcher ID

Conviene tener también presente otras iniciativas, potencialmente interesantes, creadas por otras instituciones, tales como el 'Author profile' y el 'Author Identifier', ambos desarrollados por Scopus, la base de datos creada por la poderosa Elsevier. También CrossRef, una asociación de más de dos mil editoras científicas, ha propuesto introducir un Contributor ID, un número específico para cada autor que funcionase de manera parecida a como lo hace el DOI (digital object identifiers) para documentos digitales (Qiu, 2008), a través del proyecto ORCID (Open Researcher and Contribuitor ID).

Desde la revista RELIEVE queremos contribuir a correcta identificación de nuestros autores. Por ello hace algunos años que sugerimos que las referencias de nuestros artículos deben incluir el nombre completo de los autores, en lugar de utilizar exclusivamente las iniciales, tal y como ya proponen otros estilos de citación como el MLA (2008) o el estilo Chicago -CMOS- (University of Chicago, 2010). Somos conscientes de que ello no se ajusta exactamente a la clásica normativa APA. Sin embargo, tal y como indica la sexta edición del manual APA (2010), dicha normativa no es prescriptiva, sino descriptiva de las características utilizadas en la edición de revistas científicas. A nuestro entender, utilizar el nombre completo de los autores evita confusiones entre personas que com- parten el mismo apellido, además de que el esfuerzo por incluirlo es mínimo, por lo que merece la pena esta pequeña modificación formal del formato de citación sugerido por la APA.

Como hemos podido comprobar en los párrafos anteriores, estamos en un momento histórico en el que nos estamos replanteando la mejor forma de identificar a los autores que escriben textos científicos. Son varias las posibles soluciones exploradas, la mayoría de las cuales sugerimos vivamente a los investigadores que remitan sus textos a RELIEVE.

\section{Referencias}

Aliaga, Francisco M. y Suárez-Rodríguez, Jesús M. (2007). Internacionalidad de las revistas académicas: Estudio de caso con RELIEVE. RELIEVE, v. 13, n. 1. Consultado el 15 de Diciembre de 2010http://www.uv.es/RELIEVE/v13n1/R ELIEVEv13n1 0.htm.

Correa, Ana D. y Aliaga, Francisco M. (2009). Mejora de la calidad, la gestión y la difusión de RELIEVE. RELIEVE, v. 15, n. 2. Consultado el 15 de Diciembre de 2010 en

http://www.uv.es/RELIEVE/v15n2/RELIE VEv15n2_0.htm.

Costas, R. \& Bordons, M. (2007). Algoritmos para solventar la falta de normalización de nombres de autor en los estudios 
bibliométricos. Investigación bibliotecológica: archivonomía, bibliotecología e información, 21(42), 13-32.

Delgado López-Cózar, Emilio; Ruiz-Perez, Rafael; Jiménez-Contreras, Evaristo. (2007). La Edición de Revistas Científicas: Directrices, Criterios y Modelos de Evaluación. Madrid: Fundación Española para la Ciencia y la Tecnología (FECYT).

FECYT (2007). Propuesta para la normalización del nombre de autores e instituciones en las publicaciones científicas. Consultado el 10 de Enero de 2011 en http://www.accesowok.fecyt.es/normalizac ion_nombre_autor.pdf

Goldin, Claudia \& Shim, Maria (2004). Making a Name: Surnames of College Women at Marriage and Beyond. Journal of Economic Perspectives, 18 (2), pp. 14360.

Hernández Pina, Fuensanta \& Maquilón Sánchez, Javier J. (2010). Indicadores de calidad de las revistas científicas y sistema de gestión editorial mediante OJS. Revista de investigación Educativa, vol 28 (1), 1329.

MLA (2008). MLA Style Manual and Guide to Scholarly Publishing. 3rd ed. New York: MLA.
Qiu J. (2008). Scientific publishing: identity crisis. Nature, 451:766-767. doi:10.1038/451766a

Rodríguez-Yunta, L. (2010). Las revistas iberoamericanas en Web of Science y Scopus: visibilidad internacional e indicadores de calidad. En VII Seminario HispanoMexicano de Investigación en Bibliotecología y Documentación, Ciudad de México,7-9 de abril.

Román, Adelaida (2001). La edición de revistas científicas: Guía de buenos usos. Madrid: CINDOC.

Ruiz-Pérez R, López-Cózar ED, JiménezContreras E. (2002) Spanish personal name variations in national and international biomedical databases: implications for information retrieval and bibliometric studies. $J$ Med Library Assoc, 90:411-30.

Salmerón-Pérez, Honorio; GutierrezBraojos, Calixto; Fernández-Cano, Antonio \& Salmeron-Vilchez, Purificación (2010). Aprendizaje autorregulado, creencias de autoeficacia y desempeño en la segunda infancia. RELIEVE, $v .16, n .2$. http://www.uv.es/RELIEVE/v16n2/RELIE VEv16n2_4.htm

University of Chicago (2010). The Chicago Manual of Style (16th ed.). Chicago: Univ. of Chicago Press.

\section{$\underline{\text { ABOUT THE AUTHORS / SOBRE LOS AUTORES }}$}

Aliaga, Francisco M. (Francisco.Aliaga@uv.es). Profesor Titular en el Departamento de Métodos de Investigación y Diagnóstico en Educación de la Universidad de Valencia (España). Su dirección postal es Avda. Blasco Ibáñez, 30. 46010-Valencia (España). Sus principales líneas de trabajo son "Internet y Educación", "Metodología de investigación" y "Evaluación". Fue Director Ejecutivo de RELIEVE desde 2001 hasta Junio de 2009, cuando ha sido promovido a Director. Buscar otros artículos de este autor en Scholar Google

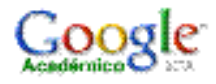

Correa, Ana D. (acorrea@ull.es). Profesora Titular en el Departamento de Didáctica e Investigación Educativa de la Universidad de La Laguna (España). Su principal línea de trabajo es "Métodos de investigación". Su dirección postal es: Campus Central, Módulo B. C/ Delgado Barreto, s/n. 38071-La Laguna (Tenerife, España). Es Directora Ejecutiva de RELIEVE desde Junio de 2009. Buscar otros artículos de esta autora en Scholar Google

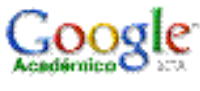




\title{
ARTICLE RECORD / FICHA DEL ARTÍCULO
}

\begin{tabular}{|c|c|}
\hline $\begin{array}{l}\text { Reference / } \\
\text { Referencia }\end{array}$ & $\begin{array}{l}\text { Aliaga, Francisco M. \& Correa, Ana D. (2011). Tendencias en la normalización de nombres de autores en } \\
\text { publicaciones científicas. RELIEVE, v. 17, n. 1. http://www.uv.es/RELIEVE/v17n1/RELIEVEv17n1_0.htm }\end{array}$ \\
\hline Title / Título & $\begin{array}{l}\text { Tendencias en la normalización de nombres de autores en publicaciones científicas. [Normalization trends } \\
\text { of authors' names in scientific publications]. }\end{array}$ \\
\hline $\begin{array}{l}\text { Authors / } \\
\text { Autores }\end{array}$ & Aliaga, Francisco M. y Correa, Ana D. \\
\hline \begin{tabular}{|l} 
Journal / \\
Revista
\end{tabular} & RELIEVE (Revista ELectrónica de Investigación y EValuación Educativa), v. 17, n. 1 \\
\hline ISSN & $1134-4032$ \\
\hline $\begin{array}{l}\text { Publication } \\
\text { date / } \\
\text { Fecha de } \\
\text { publicación }\end{array}$ & 2011 (Reception Date: 2011 March 9 [Editorial]; Publication Date: 2011 March 9). \\
\hline $\begin{array}{l}\text { Abstract / } \\
\text { Resumen }\end{array}$ & $\begin{array}{l}\text { We analyze current normalization trends of authors' names. We review the advantages of using a single } \\
\text { bibliographic name as well as the most common naming recommendations so that each author can choose } \\
\text { which to follow. } \\
\text { Analizamos las tendencias actuales sobre normalización de los nombres de autores. Se revisan las ventajas } \\
\text { de utilizar un nombre bibliográfico único y se revisan las recomendaciones más habituales para que se eli- } \\
\text { jan los de cada autor. }\end{array}$ \\
\hline $\begin{array}{l}\text { Keywords / } \\
\text { Descriptores }\end{array}$ & $\begin{array}{l}\text { Academic publishing; Internationality; Scientific communication; Impact; Citations; Author. } \\
\text { Publicación académica; Internacionalidad; Comunicación científica; Impacto; Citas; Autor }\end{array}$ \\
\hline $\begin{array}{l}\text { Institution / } \\
\text { Institución }\end{array}$ & Universidad de Valencia y Universidad de La Laguna (España). \\
\hline $\begin{array}{l}\text { Publication site } \\
\text { / Dirección }\end{array}$ & http://www.uv.es/RELIEVE \\
\hline $\begin{array}{l}\text { Language / } \\
\text { Idioma }\end{array}$ & Español \& English (Title, abstract and keywords in English \& Spanish) \\
\hline
\end{tabular}

\section{RELIEVE}

\section{Revista ELectrónica de Investigación y $\mathbf{E V}$ aluación Educativa E-Journal of Educational Research, Assessment and Evaluation}

\author{
[ISSN: 1134-4032]
}

(C) Copyright, RELIEVE. Reproduction and distribution of this articles it is authorized if the content is no modified and their origin is indicated (RELIEVE Journal, volume, number and electronic address of the document).

(C) Copyright, RELIEVE. Se autoriza la reproducción y distribución de este artículo siempre que no se modifique el contenido y se indique su origen (RELIEVE, volumen, número y dirección electrónica del documento). 